\title{
Bayesian Structured Additive Distributional Regression with an Application to Regional Income Inequality in Germany Supplement A: Case Studies
}

\author{
Nadja Klein, Thomas Kneib \\ Chair of Statistics \\ Georg-August-University Göttingen
}

\author{
Stefan Lang \\ Department of Statistics \\ University of Innsbruck
}

\author{
Alexander Sohn \\ Chair of Statistics \\ Georg-August-University Göttingen
}

\section{A.1 Supplementary Material to the Labour Income Analysis}

Data All the data is taken from the SOEP.v28 database provided by the Deutsches Institut für Wirtschaftsforschung (DIW Wagner et al., 2007). As dependent variable we consider the annual gross market labour income. We thus consider the variable LABGRO $\$$ provided in the SOEP and adjust it in accordance with Bach et al. (2009), such that the income also includes the social security contributions paid by the employer. The income is deflated using the consumer price index (Statistisches Bundesamt, 2012) and denoted in Euros at the purchasing power of the year 2010. Note that we exclude 16 observations with an annual income below $1200 €$, as this income seems improbable for a full-time employment.

Concerning education, we follow Acemoglu (2002) and use a binary education variable distinguishing between those who have received higher education and those who haven't. We use the International Standard Classification of Education which is provided in the ISCED $\$$ variable in the SOEP, defining those with lower education categorised 5 and below. Note that we use effect-coding and not dummy coding.

The East indicator is defined such that all regions in the Bundesländer that formerly belonged to the German Democratic Republic are considered as in the East. Berlin is thus also considered to belong to the East. Again we use effect coding.

For the age of the person we use the year of birth provided by the variable GEBJAHR and deduce the age from it. 


\begin{tabular}{l|l}
\hline \hline Covariate & Description \\
\hline labgrossia & $\begin{array}{l}\text { gross market labour income } \\
\text { (continuous, } 1,226 € \leq \text { labgrossia } \leq 1,402,000 € \text {, average }=50,200 €)\end{array}$ \\
educ & $\begin{array}{l}\text { education (binary, }-1=\text { low education }(79.6 \%), 1=\text { high education }(20.4 \%)) \\
\text { East }\end{array}$ \\
age & $\begin{array}{l}\text { age of the male in years (continuous, } 20 \leq \text { age } \leq 60, \text { average }=42) \\
\text { labour market experience in years }\end{array}$ \\
$\mathrm{t}$ & $\begin{array}{l}\text { (continuous, } 0 \leq \text { lmexp } \leq 45.1, \text { average }=19) \\
\text { time in years (categorical, 2001 } \leq \mathrm{t} \leq 2010,10 \text { years, average }=2005)\end{array}$ \\
$\mathrm{s}$ & district (Raumordnungsregion) (categorical, 96 districts) \\
\hline \hline
\end{tabular}

Table A1: German labour income data. Description of covariates.

For the labour market experience both full-time (EXPFT $\$$ ) and part-time (EXPPT $\$$ ) experience alike, simply taking the sum of the two variables.

The information on the district is taken from the variable ROR96\$ included in the SOEP-Geocode database.

All observations are weighted using the weights provided in the variable $\$ \mathrm{PHRF}$. We thus use the cross sectional weights and not the longitudinal weights which include information on attrition. Consequently, our panel is not balanced.

Quantile Residuals of Models of Type M2 and M3 The quantile-quantile plots of quantile residuals for the models of type M2 and M3 are shown in Figure A1. As mentioned in the main paper, differences compared to models M1 in Figure 2 are rarely visible such that residuals in general indicate that the Dagum distribution fits best.

Probability Integral Transforms of Models of Type M1 Probability integral transforms for the models of type M1 are given in Figure A2. Similar to the quantile residuals in Figure 2 of the main paper they indicate that the Dagum distribution gives the best fit.

Decomposition of Scores A decomposition of the scores (averages are documented in Table 2 of the main paper) over the cross validation folds is given in Figure A3. The plot with separate scores per fold shows a homogeneous average behaviour of the scores across the folds. This provides a basic check for structural differences in the folds which may arise in disadvantageous random assignments. 

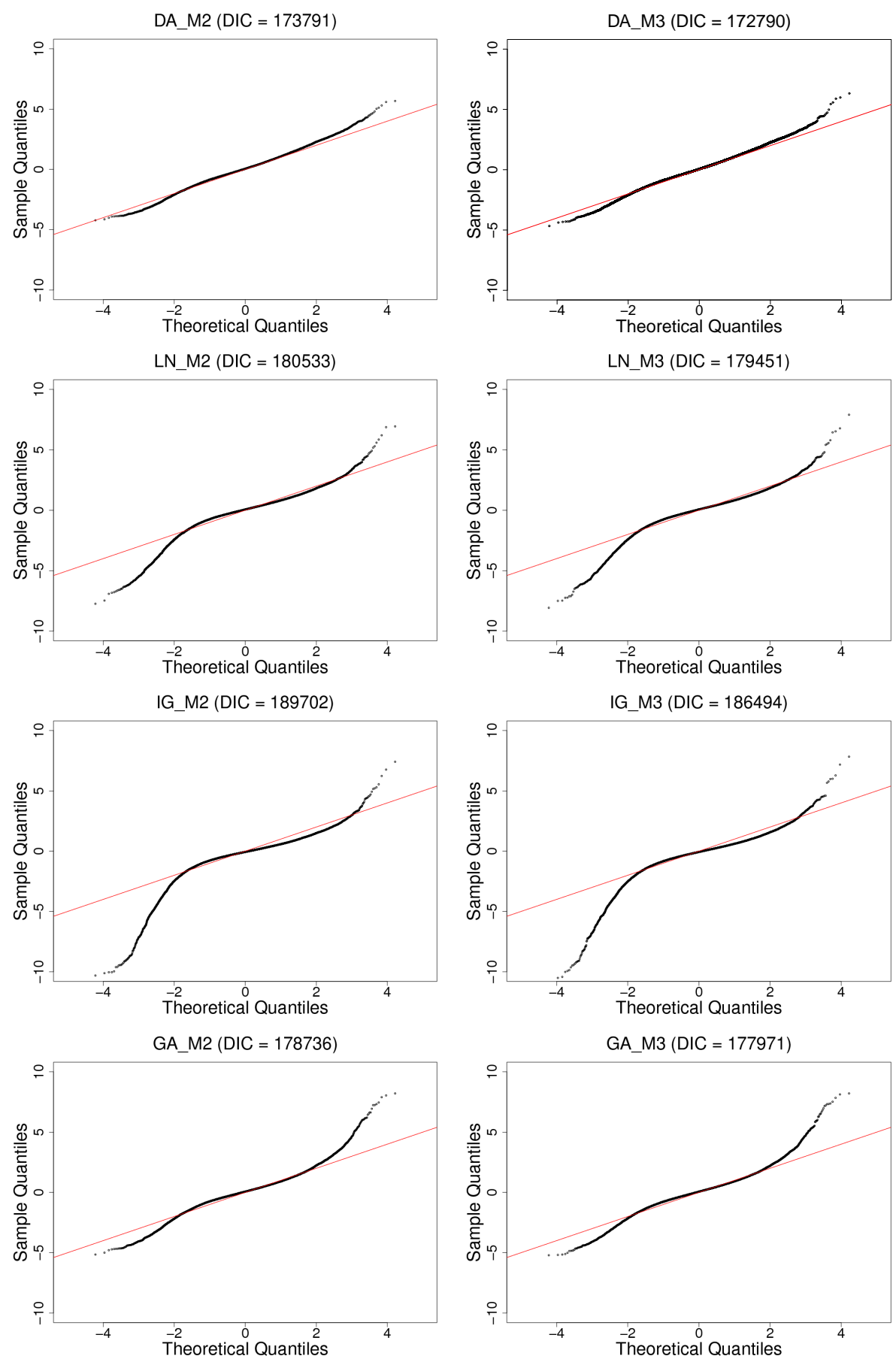

Figure A1: SOEP data. Comparison of quantile residuals for the models DA_M2, DA_M3 (first row), LN_M2, LN_M3 (second row), IG_M2, IG_M3 (third row), GA_M2, GA_M3 (fourth row). Models M2 correspond to the ones in which the parameters $\sigma, \sigma^{2}, a, c$ are estimated as constants. In models M3 the random effects for year and region have been removed compared to M1.

\section{A.1.1 Further Analysis of the Conditonal Income Distributions}

As we have demonstrated in Section 4, we can assess conditional income distributions using structured additive distributional regression. For reasons of brevity, we only 
DA_M1 $(D I C=172421)$

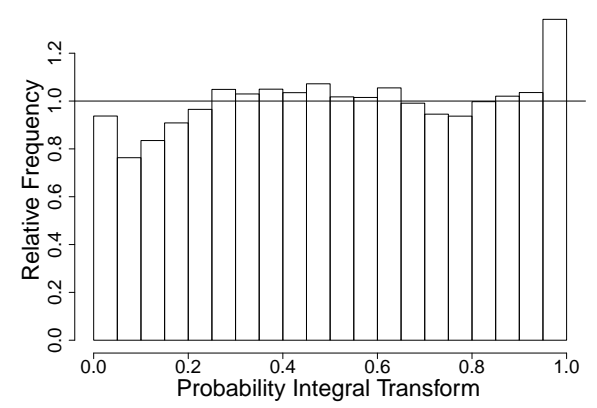

IG_M1 (DIC = 184614)

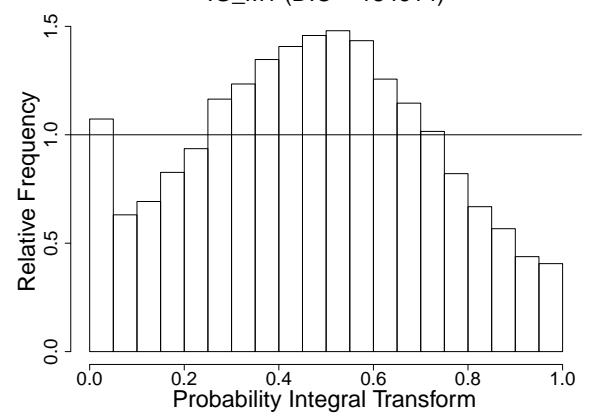

LN_M1 $(D I C=179090)$

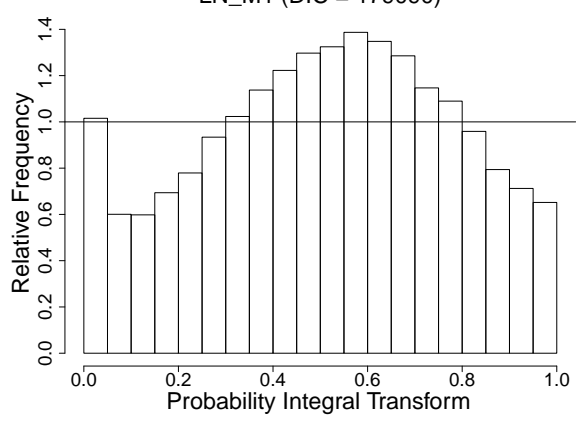

GA_M1 $(D I C=177453)$

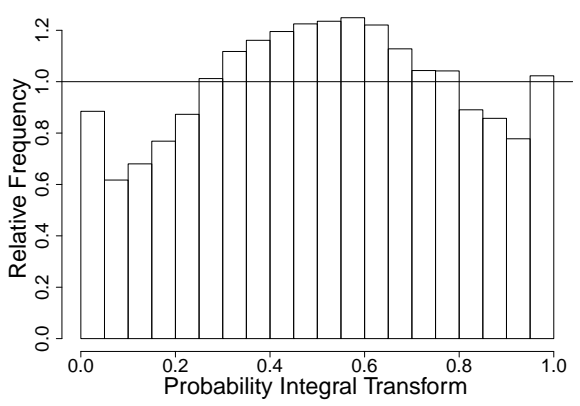

Figure A2: Comparison of probability integral transforms for the full models DA_M1 (topleft), LN_M1 (topright), IG_M1 (bottomleft), GA_M1 (bottomright) (see Section 3).

provided a very narrow analysis in the text, focussed on the divide between East and West Germany. Of course, it is also possible to provide estimates for other covariates combinations. While the number of dimensions makes a comprehensive analysis of all the possible combinations intractable, we will consider the effect of varying each covariate on the conditional mean and the conditional Gini coefficient, while keeping the other continuous covariates fixed at their mean and categorical variables at the mode.

\section{The Effect of Varying the Years on the Conditional Income Distribution}

In our data we include a ten-year period and model the different years as random effects. The impact of these random effects on the mean income and the Gini-coefficient given that the other covariates are set at their mean or for the categorical variables at the mode is displayed in Figure A4

For the mean, we can observe that we have a slight decline over the years, which is not significant though. Nonetheless, the decline of labour incomes over the first decade of the new millenium is in line with the falling labour incomes in many sectors of the economy and the falling share of labour incomes in the gross added value.

Concerning the Gini coefficient, we also see no significant change over the ten years. This would indicate that the rising income inequality over the years is driven by 

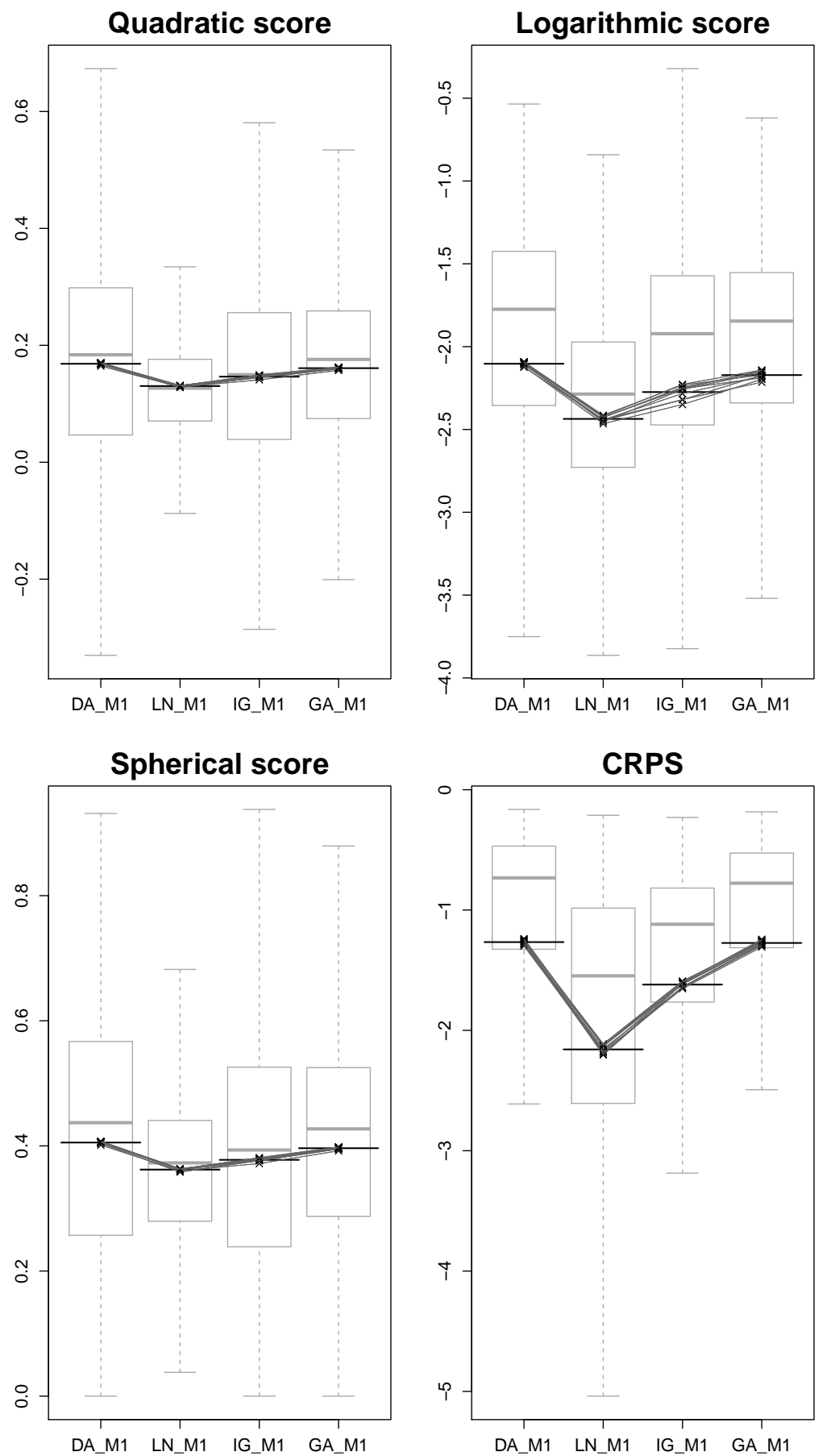

Figure A3: SOEP data. Boxplots (outliers exceeding 1.5 times the interquartile range are omitted) of individual score contributions for the whole data set. Preditions have been obtained by ten fold cross validation. The black crosses indidate average scores in each fold and are connected by grey lines between the four models. The models under consideration in each graph are DA_M1, LN_M1, IG_M1, GA_M1 (on the x-axes). On the y-axes values of the quadratic score (topleft), logarithmic score (topright), spherical score (bottomleft) and the CRPS (bottomright) are shown. Grey lines in the boxes are the median scores, the black lines the average scores documented in Table 2 

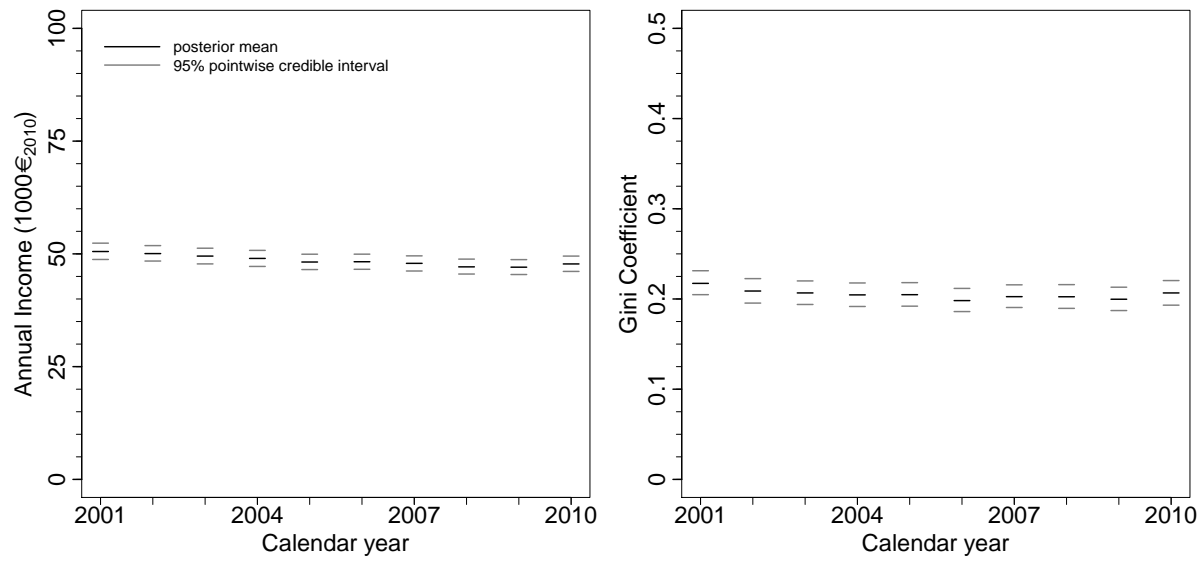

Figure A4: SOEP data. Posterior means for the expected incomes (left) and Gini coefficients (right) for 42-year-old males with 19 years of working experience, without higher education and living in the West, together with $95 \%$ pointwise credible intervals.

changes between groups not within them. Again it must be noted that this relies on a rather rigid model specification of constant temporal effects on the predictors over the whole covariate space. 


\section{Without Higher Education}

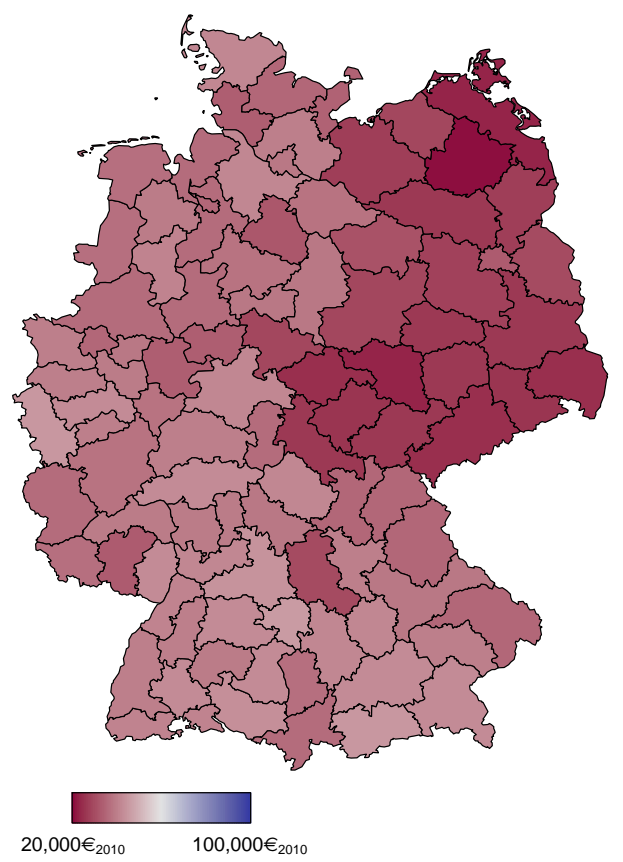

With Higher Education

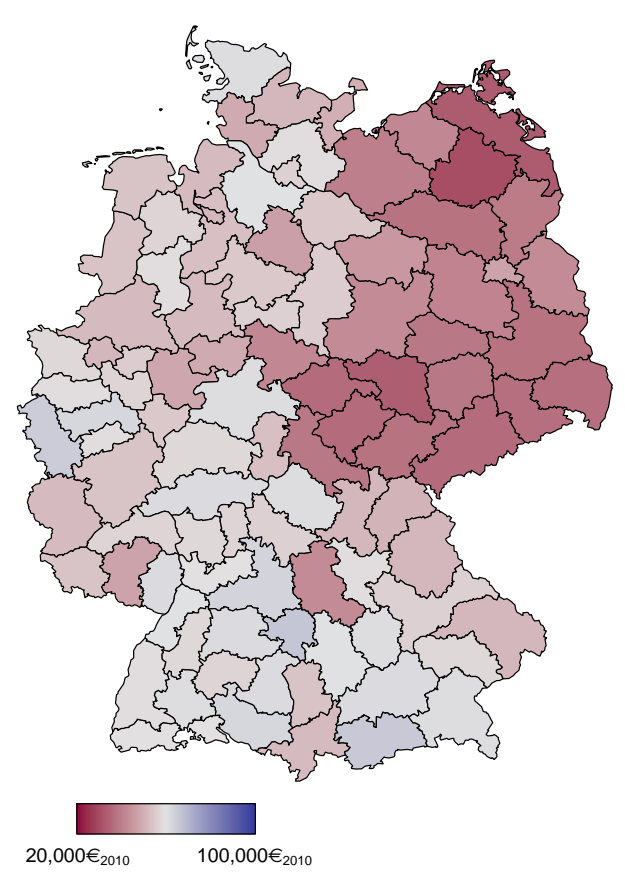

Figure A5: SOEP data. Posterior means for the expected incomes for 29-year-old males with 5 years of working experience in the year 2001. Left: males without higher education. Right: males with higher education.

\section{A.1.2 The Spatial Effect for Three Alternative Covariate Combinations}

Here we consider three alternative sets of covariate values compared to our considered setting in the main paper (compare Section 4) and the effect that this has on the differences of conditional incomes.

Set 1 In this covariate set, we consider the spatial effect for a male person with age 29 and 5 years of work experience, which constitutes the $10^{\text {th }}$ percentile of the empirical cumulative distribution functions of the two variables. The covariate year is set to 2001. The resultant posterior means for the expected incomes are displayed in Figure A5, If we centre the regional random effect at zero, the resultant difference in the posterior mean of the average incomes for lower education between East and West is $12,200 €$ with the corresponding $95 \%$ credible interval at $[10,100 € ; 14,300 €]$. For higher education the difference is $17,600 €[14,700 € ; 20,700 €]$.

For the standard deviations, the posterior means for the regions are displayed in Figure A6. Abstracting the random effects, the difference between East and West is $2,600 €[1,400 € ; 3,700 €]$ and $4,000 €[2,100$ euro;5,800€] for lower and higher education respectively.

For the Gini coefficients, the posterior means for the regions are displayed in Figure 


\section{Without Higher Education}

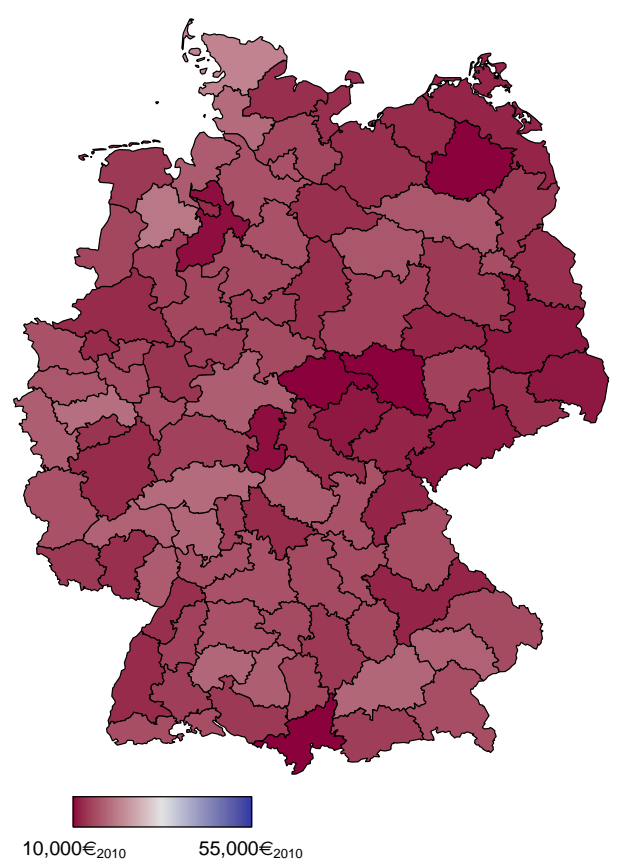

With Higher Education

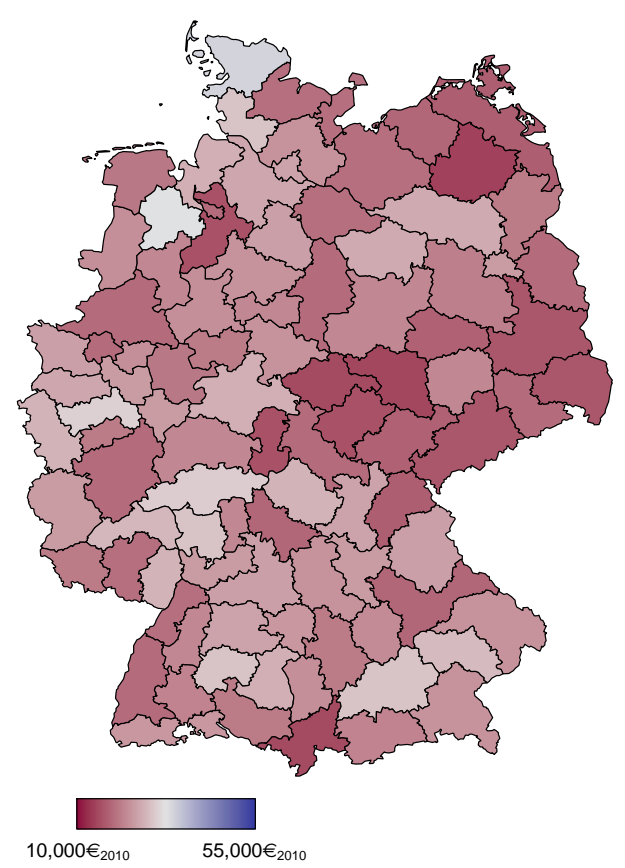

Figure A6: SOEP data. Posterior means for the standard deviations for 29-year-old males with 5 years of working experience in the year 2001. Left: males without higher education. Right: males with higher education.
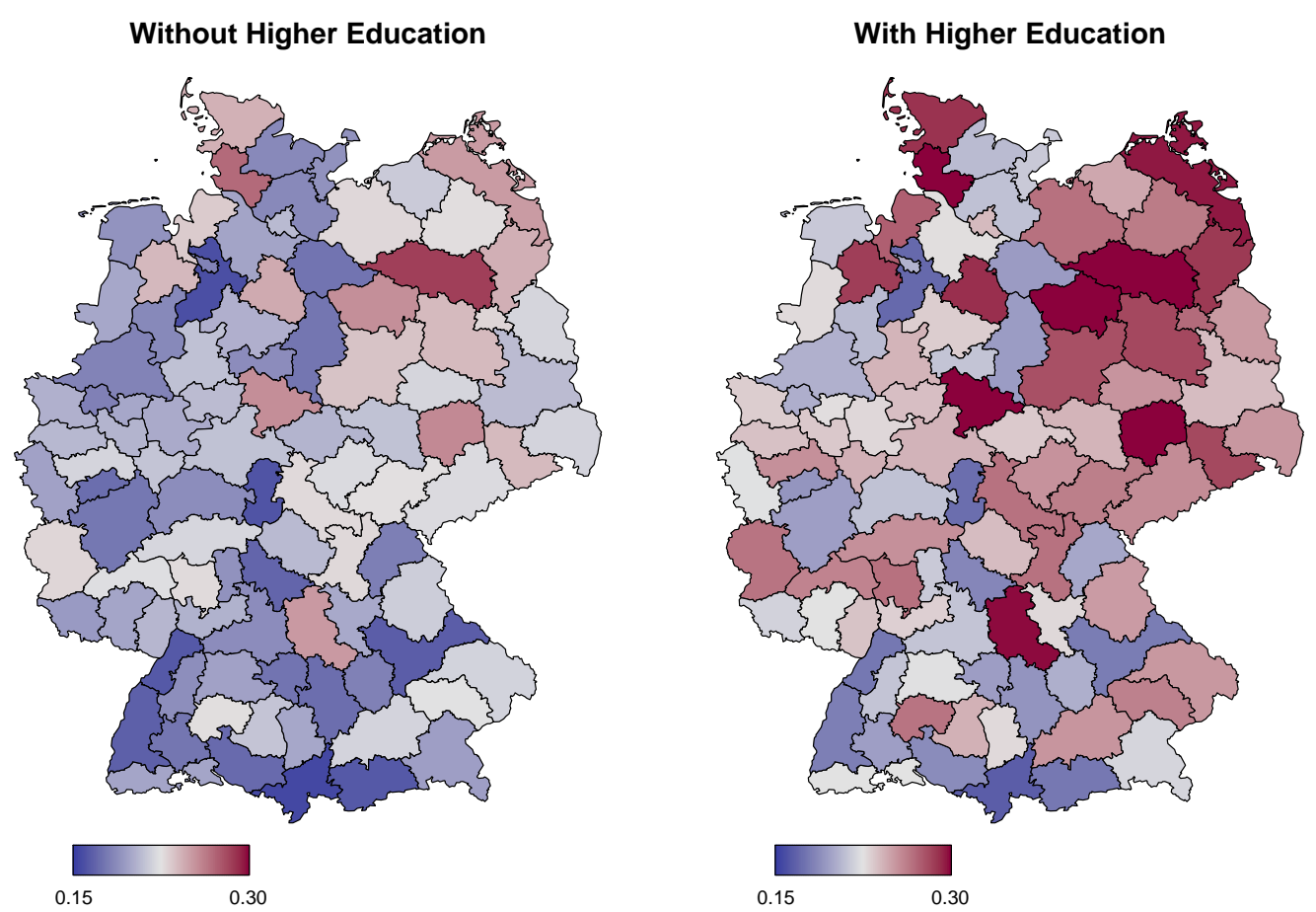

Figure A7: SOEP data. Posterior means for the Gini coefficients for 29-year-old males with 5 years of experience in the year 2001. Left: males without higher education. Right: males with higher education.

A7. Again abstracting the random effects, the difference between East and West is 
Without Higher Education

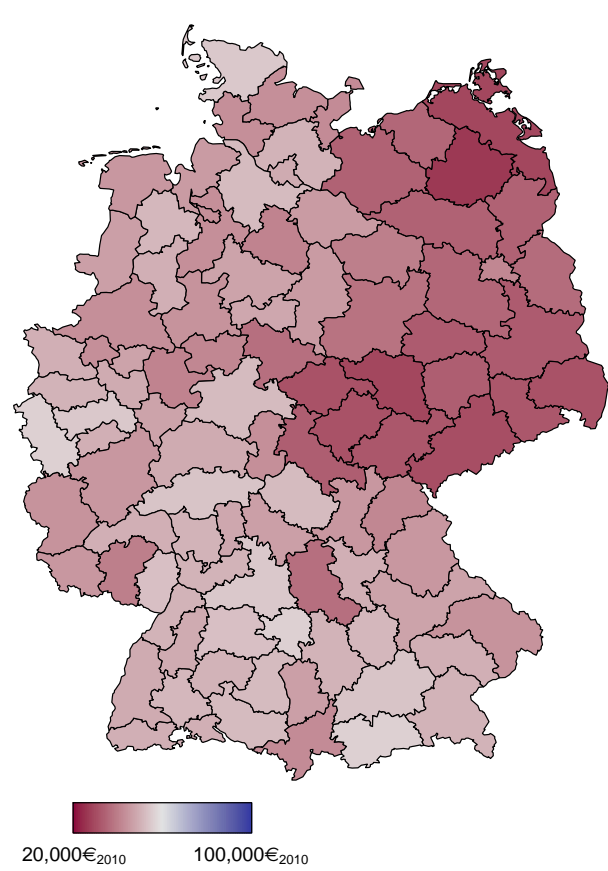

With Higher Education

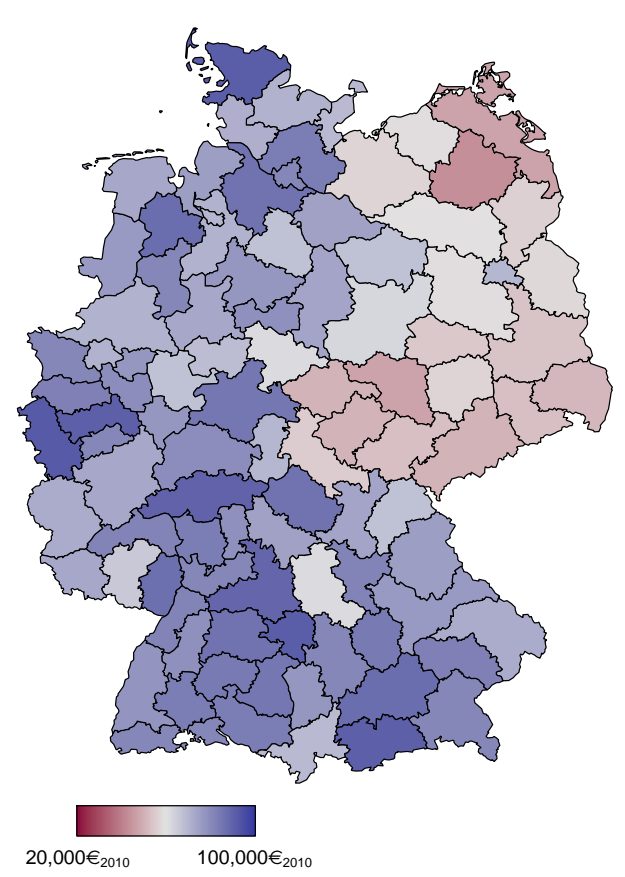

Figure A8: SOEP data. Posterior means for the expected incomes for 42-year-old males with 19 years of working experience in the year 2005. Left: males without higher education. Right: males with higher education.

$0.044[0.019,0.074]$ and $0.045[0.019,0.076]$ for lower and higher education respectively.

Set 2 In this covariate set, we consider the spatial effect for a male person with age 42 and 19 years of work experience, which constitutes the $50^{\text {th }}$ percentile of the empirical cumulative distribution functions of the two variables. The covariate year is set to 2005. The resultant posterior means for the average incomes are displayed in Figure A8, If we centre the regional random effect at zero, the resultant difference in the posterior mean of the average incomes for lower education between East and West is $14,500 €$ with the corresponding credible interval at $[12,000 € ; 16,800 €]$. For higher education the difference is $23,100 €[19,100 € ; 27,200 €]$.

For the standard deviations, the posterior means for the regions are displayed in Figure A9. Abstracting the random effects, the difference between East and West is $3,200 €[1,300 € ; 5,200 €]$ and $5,300 €[1,700 € ; 8,700 €]$ for lower and higher education respectively.

For the Gini coefficients the posterior means for the regions are displayed in Figure A10. Again abstracting the random effect, the difference between East and West is $0.039[0.015,0.067]$ and $0.037[0.013,0.063]$ for lower and higher education respectively. 


\section{Without Higher Education}

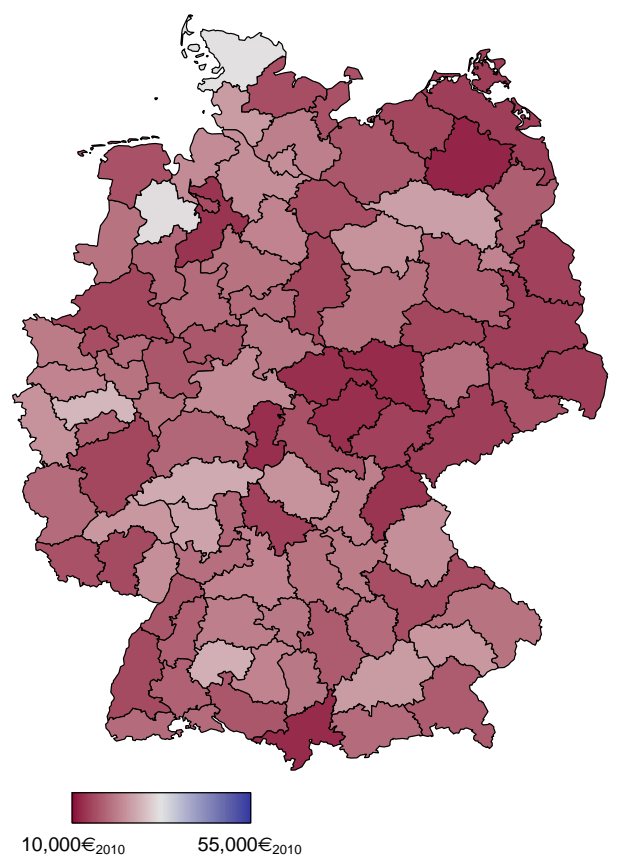

With Higher Education

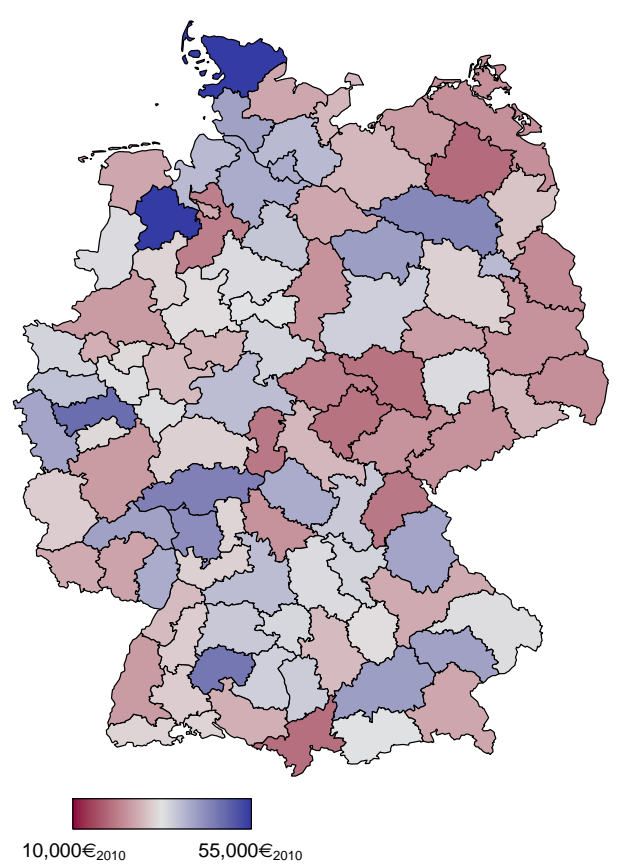

Figure A9: SOEP data. Posterior means for the standard deviations for 42-year-old males with 19 years of working experience in the year 2005. Left: males without higher education. Right: males with higher education.
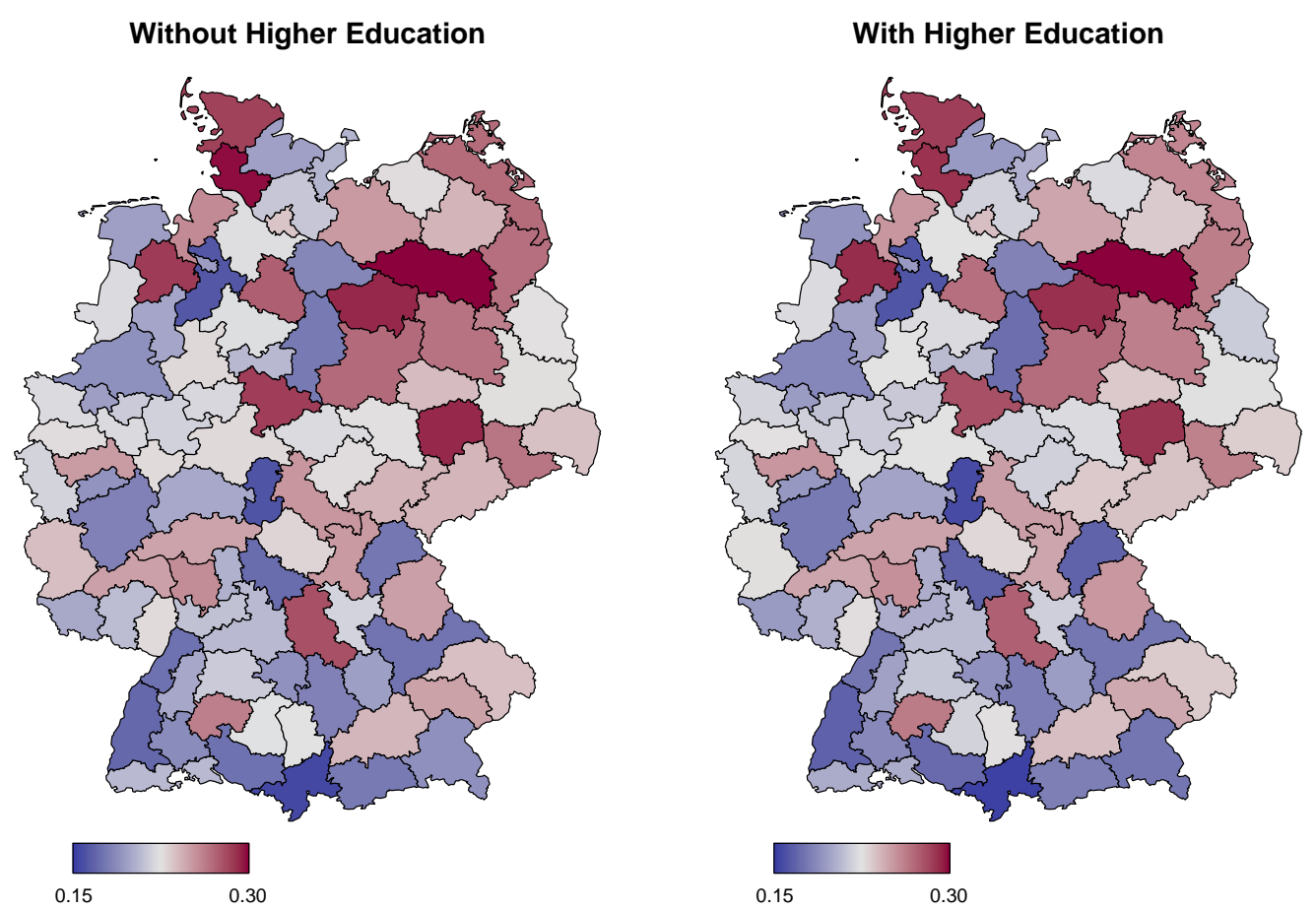

Figure A10: SOEP data. Posterior means for the Gini coefficients for 42-year-old males with 19 years of experience in the year 2005. Right: males with higher education.

Set 3 In this covariate set, we consider the spatial effect for a male person with age 49 and 34 years of work experience, which constitutes the $90^{\text {th }}$ percentile of the 
Without Higher Education

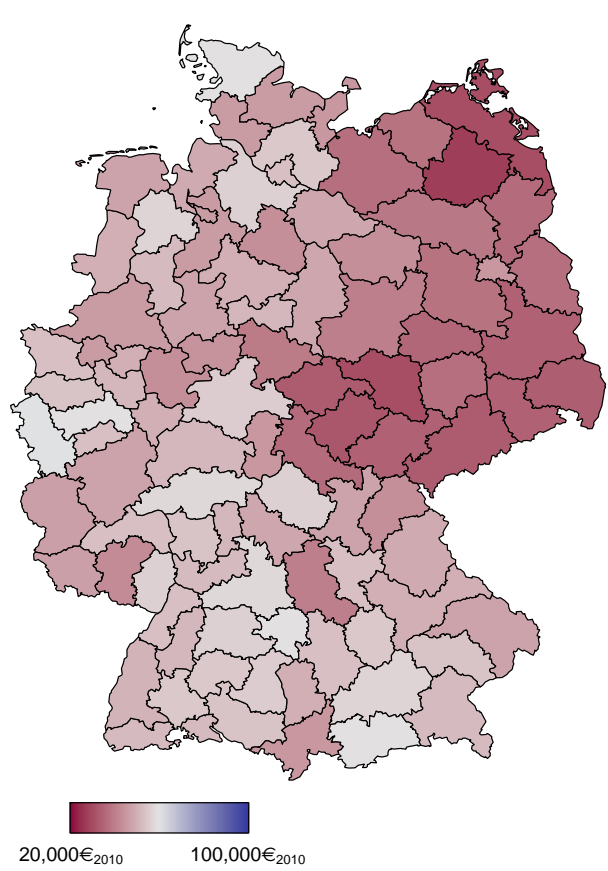

With Higher Education

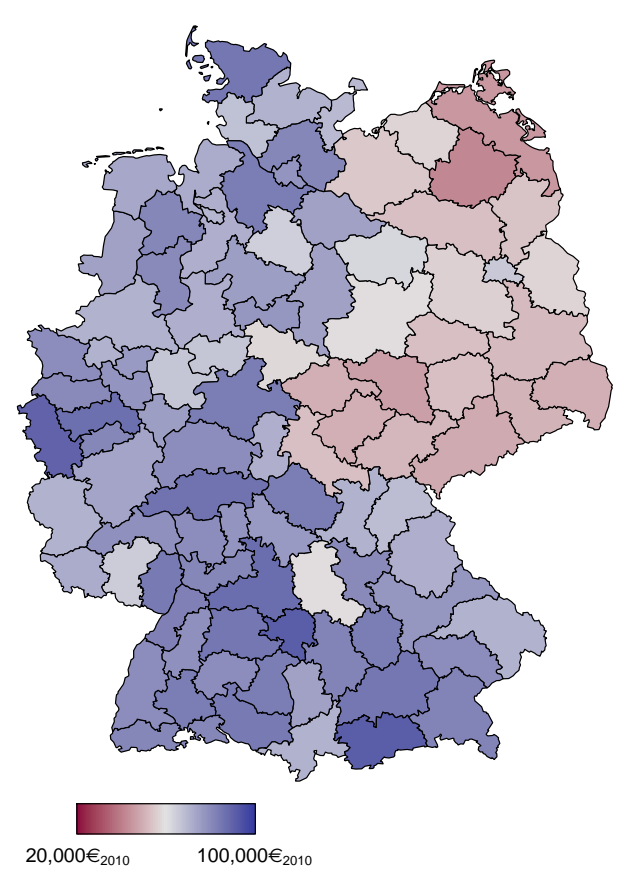

Figure A11: SOEP data. Posterior means for the expected incomes for 49-year-old males with 34 years of working experience in the year 2010, together with $95 \%$ simultaneous credible bands. Left: males without higher education. Right: males with higher education.

empirical cumulative distribution functions of the two variables. The covariate year is set to 2010. The resultant posterior means for the average incomes are displayed in Figure A11, If we centre the regional random effect at zero, the resultant difference in the posterior mean of the average income for lower education between East and West is $15,100 €$ with the corresponding credible interval at $[12,500 € ; 17,700 €]$. For higher education the difference is $24,100 €[19,800 € ; 28,200 €]$.

For the standard deviations, the posterior means for the regions are displayed in Figure A12.

Abstracting the random effect, the difference between East and West is $3,700 €[1,200 € ; 5,900 €]$ and $5,900 €[3,100 € ; 8,800 €]$ for lower and higher education respectively.

For the Gini coefficients the posterior means for the regions are displayed in Figure A13. Again abstracting the random effects, the difference between East and West is $0.039[0.014,0.067]$ and $0.046[0.019,0.077]$ for lower and higher education respectively.

Summary Overall, we find that while the magnitude of the differences between East and West varies for the mean income, standard deviation and the Gini coefficient, 


\section{Without Higher Education}

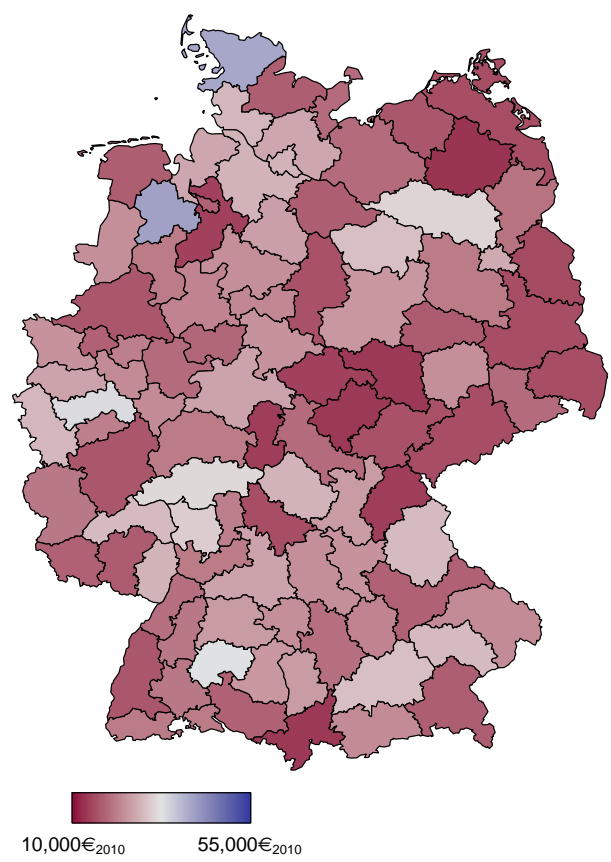

With Higher Education

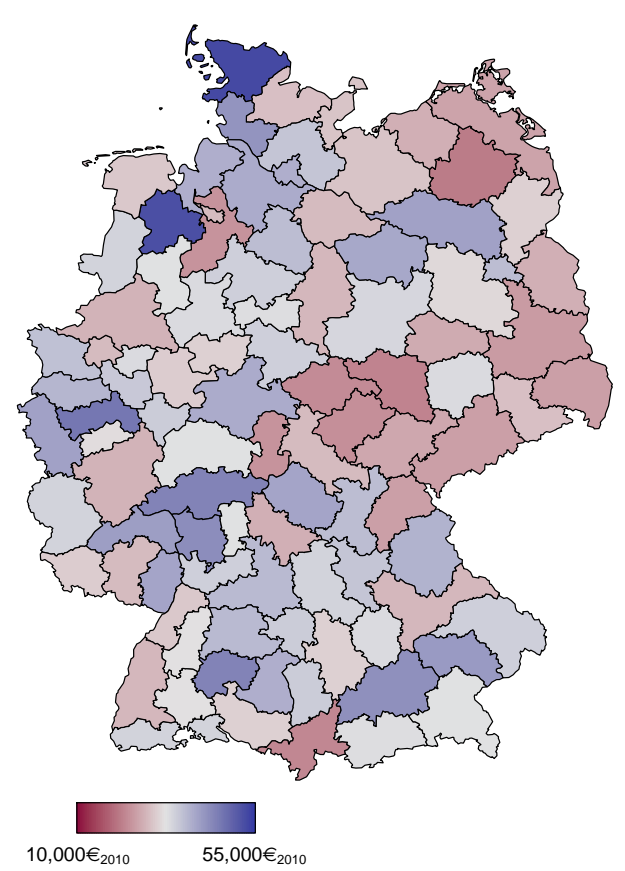

Figure A12: SOEP data. Posterior means for the standard deviations for 49-year-old males with 34 years of working experience in the year 2010, together with $95 \%$ simultaneous credible bands. Left: males without higher education. Right: males with higher education.
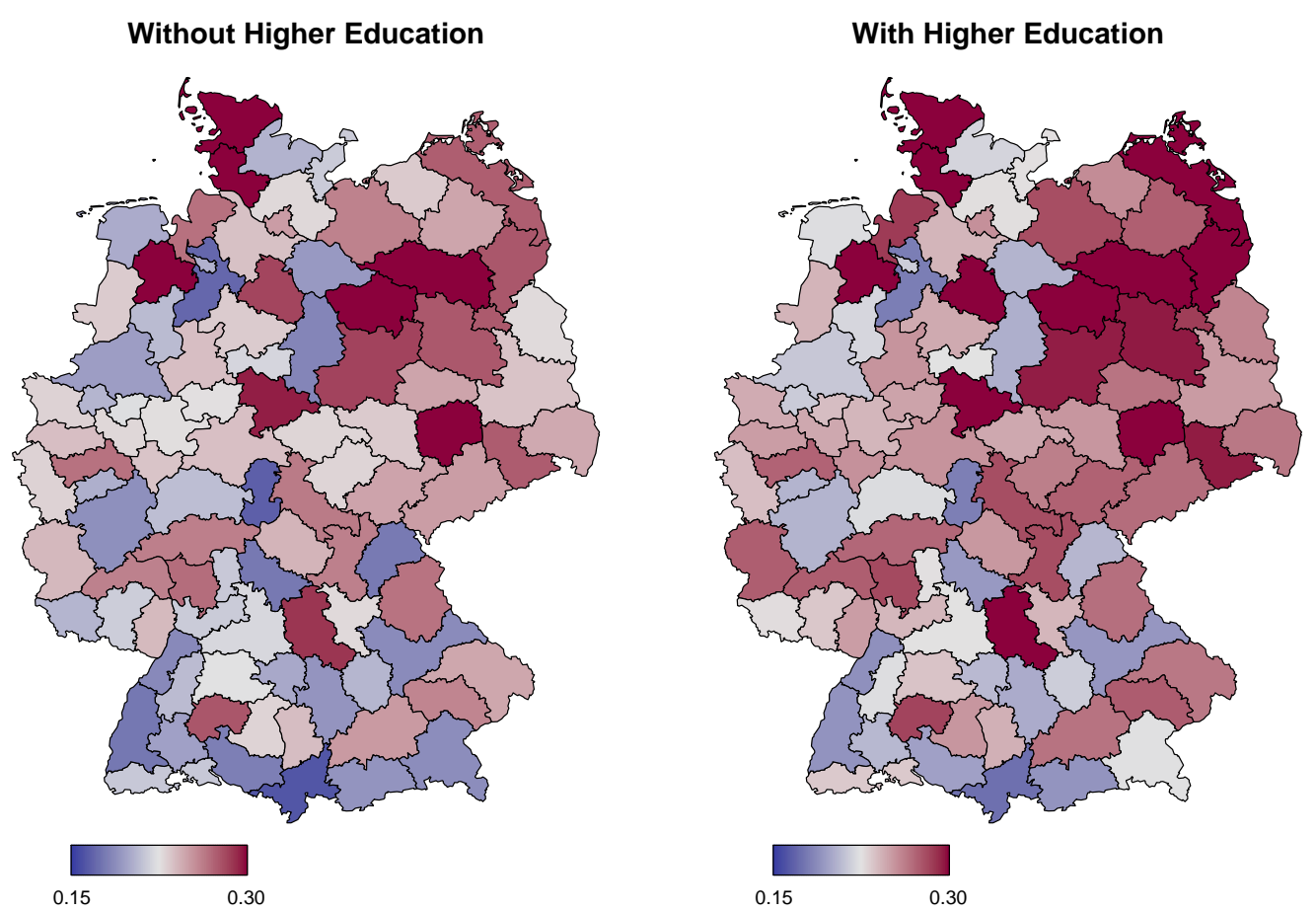

Figure A13: SOEP data. Posterior means for Gini coefficients for 49-year-old males with 34 years of experience in the year 2010. Right: males with higher education.

the direction and the significance of the effect at the $5 \%$ level remains persistent. Although further testing on the issue is required, we can thus assume that these 
differences are not an artefact of the covariate set selected. Thus, we show systematic differences not only for the conditional mean but also for the conditional spread and the conditional inequality between East and West. 


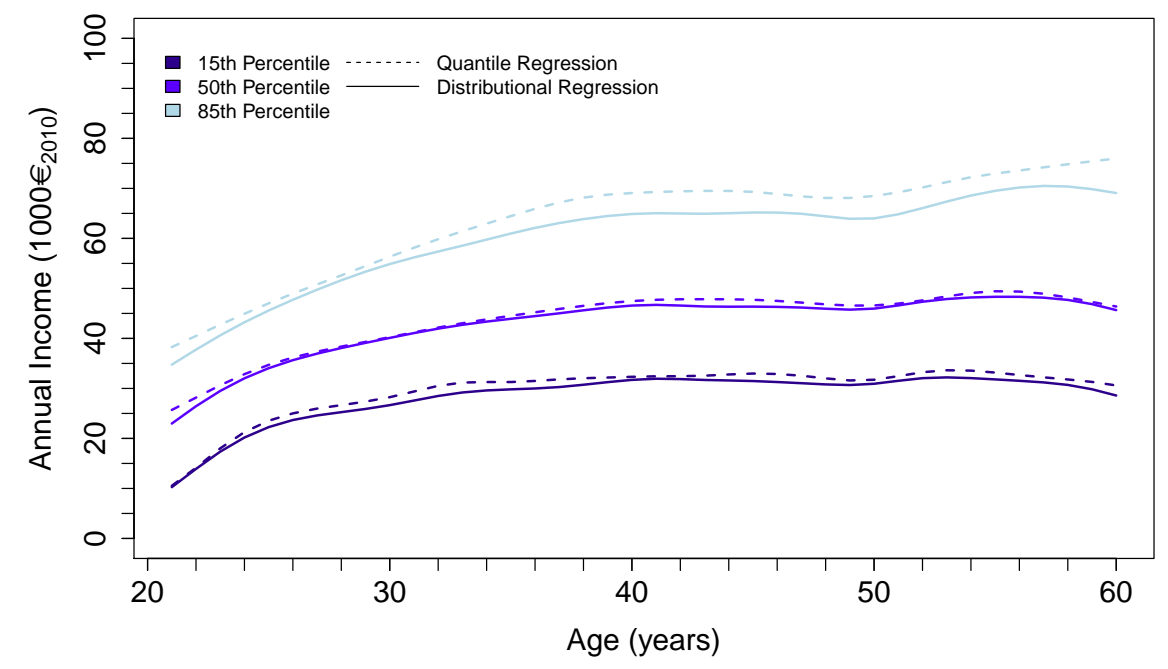

Figure A14: SOEP data. Comparsion of distributional regression (solid lines) and quantile regression (dashed lines). Shown are estimated posterior means of $15 \%, 50 \%$ and $85 \%$ quantiles of the conditional income distributions for males without higher education and living in the West.

\section{A.1.3 Comparison with Quantile Regression}

In this section, we compare estimates obtained by structured additive quantile regression (Waldmann et al., 2013) with the results we obtain from our distributional regression approach. For quantile regression, we use the same covariate effects as for our distributional regression. Since we have a multiplicative impact of the variables in our distributional set-up, we consider the logarithm of the income as a dependent variable. For the $\tau$-th quantile we thus consider

$$
\begin{aligned}
\log \left(\text { labgrossia }_{i}\right)= & \beta_{0, \tau}+\operatorname{educ}_{i} \beta_{1 \tau}+f_{1, \tau}\left(\text { age }_{i}\right)+\operatorname{educ}_{i} f_{2, \tau}\left(\text { age }_{i}\right) \\
& +f_{3, \tau}\left(\operatorname{lmexp}_{i}\right)+f_{\text {spat }, \tau}\left(s_{i}\right)+f_{\text {time }, \tau}\left(t_{i}\right)+\varepsilon_{i, \tau}
\end{aligned}
$$

with error terms $\varepsilon_{i, \tau}$, compare Waldmann et al. (2013) for further details.

Again we can only consider one variable at a time and will keep all other covariates at their mean or mode, as done previously. Following Autor et al. (2008) we consider the $15^{\text {th }}, 50^{\text {th }}$ and $85^{\text {th }}$ percentile of the conditional income distribution, i.e. $\tau=$ $0.15,0.5,0.85$.

Figure A14 displays the joint effect of age and experience (as above), while keeping the other covariates fixed. As we can see, our distributional regression approach shows a similar pattern as quantile regression. All the differences are within the bounds of estimation uncertainty. The only slightly larger difference for the $85^{\text {th }}$ percentile 


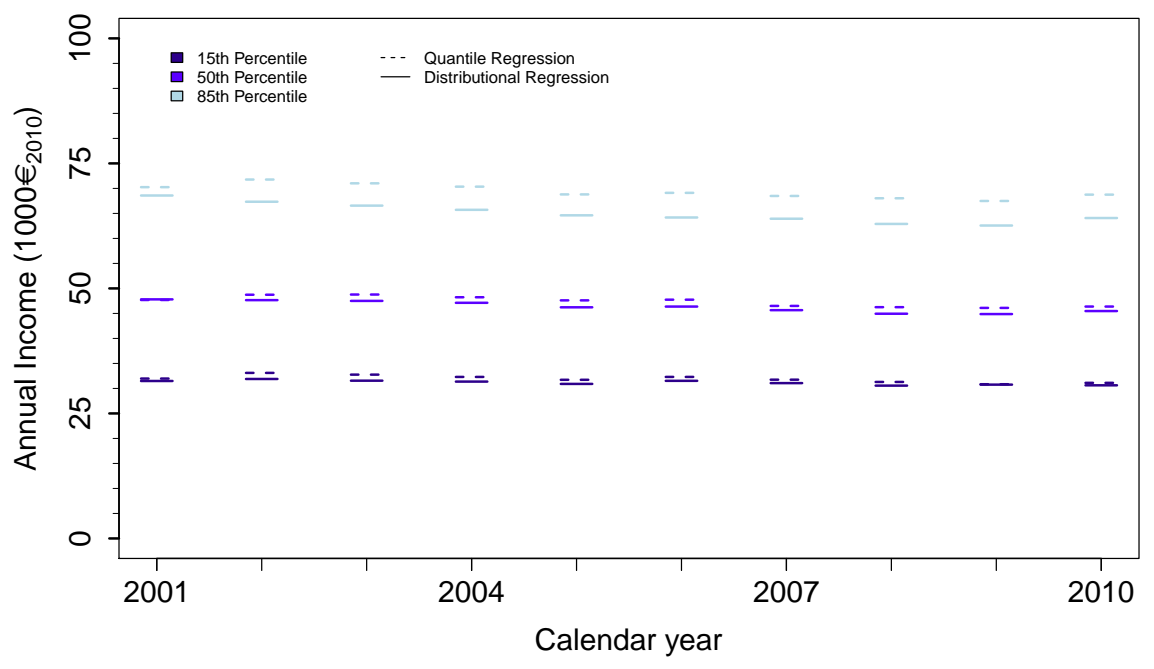

Figure A15: SOEP data. Comparsion of distributional regression (solid lines) and quantile regression (dashed lines). Shown are estimated posterior means of 15\%, 50\% and $85 \%$ quantiles of the conditional income distributions for 42-year-old males with 19 years of working experience, without higher education and living in the West.

can mostly likely be ascribed to the fact that the distributional assumption imposes slightly lighter tails than we observe in the empirical distribution of the sample. Figure $\mathrm{A15}$ shows the effect of random effects for the calender year. Both the $15^{\text {th }}$ and the $50^{\text {th }}$ percentile are similar if estimated with distributional and quantile regression. For the $85^{\text {th }}$ percentile the deviations are slightly larger. Quantile regression yields estimates that are higher than those obtained from distributional regression. The most likely reasons for the differences are analogue to those discussed above.

Concerning the spatial effect, which is displayed in Figure A16, we can see that there are again slight differences but with very similar tendencies. 

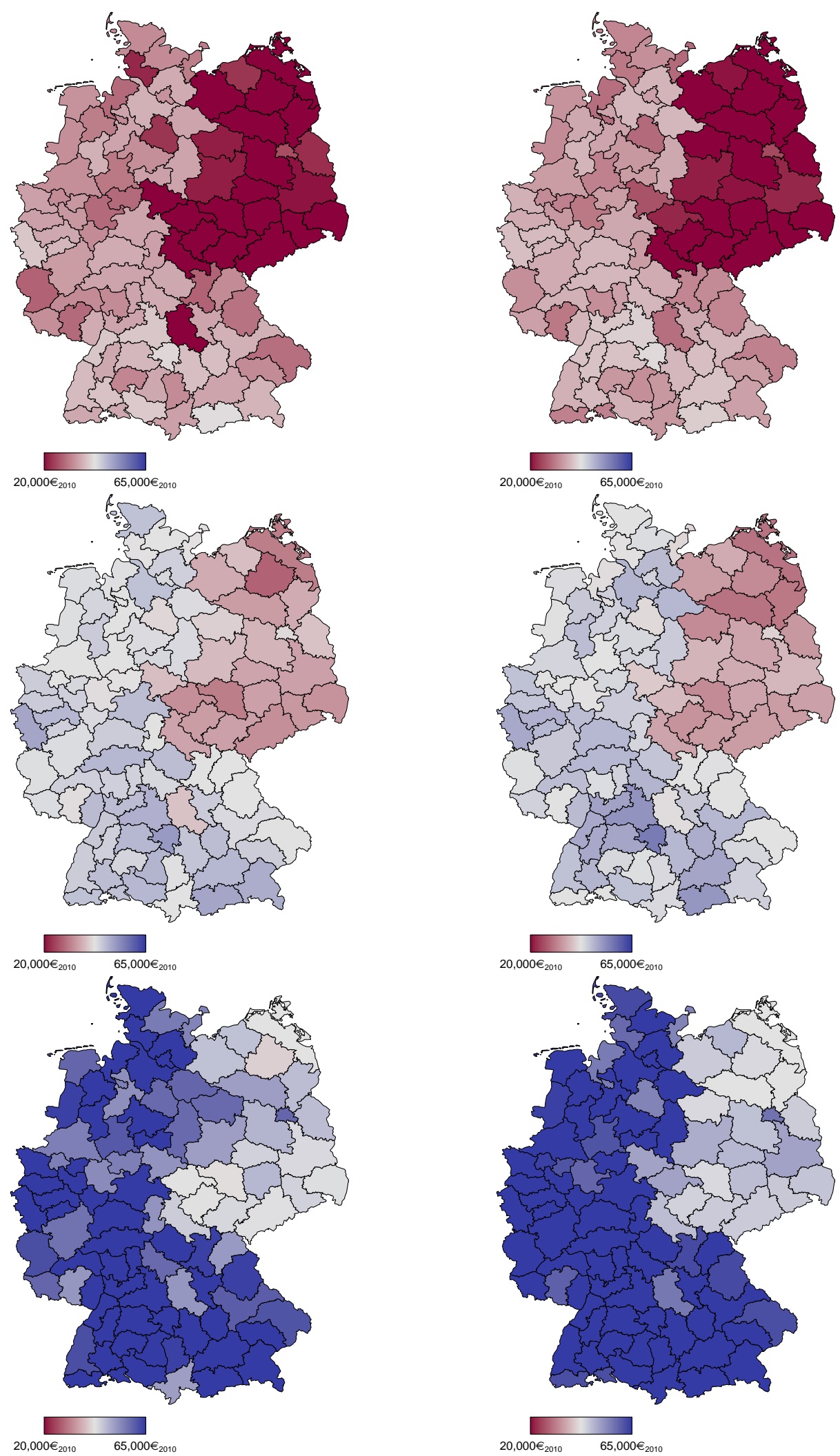

Figure A16: SOEP data. Comparsion of distributional regression (left) and quantile regression (right). Shown are estimated posterior means of $15 \%$ (top), $50 \%$ (middle) and $85 \%$ (bottom) quantiles of the conditional income distributions for 42-year-old males with 19 years of working experience, without higher education and living in the West. 


\section{A.2 Production of Cereals in Farms of England and Wales}

As a second illustration of Bayesian distributional regression, we consider the output proportions produced by the cultivation of cereal (including e.g. wheat, rice, maize) of 1232 different farms in England and Wales in the year 2007. The data have been collected by the Department of Environment, Food and Rural Affairs and National Assembly for Wales, Farm Business Survey, 2006-2007, and are provided by the UK Data Service (Colchester, Essex: UK Data Archive, 2008, http://dx.doi.org/10.5255/UKDA-SN-5838-1). The total output of the farms is subdivided in cereals and several animal products and if a farm reports positive output of cereals this can either be used for internal purposes (e.g. feeding of animals) or for selling.

Hence, the response variable is restricted to the interval $[0,1]$ and represents the amount of cereal products by the farms relative to their total output. A natural candidate for analysing such ratios is the beta distribution parametrised such that one parameter represents the expectation while the other one relates to a general shape parameter (see for example Ferrari and Cribari-Neto, 2004). However, a further complication arises in our data set from the fact that there is a considerable fraction of observations with either no production of cereal at all or complete specialisation on cereal (cereal output equal to 100\%) such that the beta distribution has to be inflated with zeros and ones. Specifically, around $55 \%$ of the farms do not produce any cereal, $5 \%$ are completely specialised on the cultivation of cereal (100\% of output are cereals) and the remaining farms have an output that is based on cereal and animal production. Therefore, the zero-one inflated beta distribution with parameters $\mu, \sigma^{2}, \nu$ and $\tau$ as described in Section B.1.1 is an appropriate candidate for analysing output shares on the cultivation of cereal. Note that in this mixed discrete-continuous distribution set-up, none of the four parameters is directly related to location scale and shape, supporting our choice of the term distributional regression as compared to GAMLSS. Note also that the structure of the response variable in this example renders both quantile regression and transformations of the response distribution inappropriate due to the mixture of a discrete and a continuous part.

From the list of potential explanatory variables related to farm business, we focus on the capital of the farms measured in pounds by their machinery, buildings and land maintenance, as well as running costs and the sum of the annual family hours of labour and the annual hired hours of labour. Furthermore, we consider the utilised 
agricultural area in hectare and to capture spatial variations, we also take into account the geographic information on the location of the farms in one of the counties in England and Wales. A generic predictor for farm $i$ can thus be written as

$$
\eta_{i}=\beta_{0}+f_{1}\left(\text { lland }_{i}\right)+f_{2}\left(\text { llabour }_{i}\right)+f_{3}\left(\text { lcapital }_{i}\right)+f_{\text {spat }}\left(\text { count }_{i}\right)
$$

where $\beta_{0}$ represents the overall level of the predictor, $f_{1}$ to $f_{3}$ are nonlinear functions of logarithmic land size (lland), the logarithm of capital (lcapital), as well as the logarithmic labour (llabour) modelled by cubic P-splines with 20 inner knots and second order random walk prior, and the spatial effect $f_{\text {spat }}$ is based on a Markov random field prior.

After comparing different models in terms of DIC, we found that several covariates have linear influences on $\sigma^{2}, v$ and $\tau$ and ended up with the following predictor structures for the four distribution parameters:

$$
\begin{aligned}
\eta_{i}^{\mu} & =\beta_{0}^{\mu}+f_{1}^{\mu}\left(\text { lland }_{i}\right)+f_{2}^{\mu}\left(\text { llabour }_{i}\right)+f_{3}^{\mu}\left(\text { lcapital }_{i}\right)+f_{\text {spat }}^{\mu}\left(\text { count }_{i}\right) \\
\eta_{i}^{\sigma^{2}} & =\beta_{0}^{\sigma^{2}}+\text { lland }_{i} \beta_{1}^{\sigma^{2}}+f_{2}^{\sigma^{2}}\left(\text { llabour }_{i}\right)+\text { lcapital }_{i} \beta_{3}^{\sigma^{2}}+f_{\text {spat }}^{\sigma^{2}}\left(\text { county }_{i}\right) \\
\eta_{i}^{v} & =\beta_{0}^{v}+f_{1}^{v}\left(\text { lland }_{i}\right)+f_{2}^{v}\left(\text { llabour }_{i}\right)+f_{3}^{v}\left(\text { lcapital }_{i}\right)+f_{\text {spat }}^{v}\left(\text { county }_{i}\right) \\
\eta_{i}^{\tau} & =\beta_{0}^{\tau}+\text { lland }_{i} \beta_{1}^{\tau}+\text { llabour }_{i} \beta_{2}^{\tau}+f_{\text {spat }}^{\tau}\left(\text { count }_{i}\right) .
\end{aligned}
$$

Figure A17 shows posterior mean estimates for the probabilities $\frac{v}{1+v+\tau}$ and $\frac{\tau}{1+v+\tau}$ as well as posterior means for the expectation $\frac{\mu+\tau}{1+v+\tau}$ of the response which correspond to the probability of exclusively and no cereal output or to the expected proportion of the total output produced by cereal, varying over the counties. All other covariates are kept constant at the estimates obtained for average covariate values. The influence of the nonlinear effects lland, llabour and lcapital on the expectation of the response is given in Figure A18, where posterior mean estimates of $\frac{\mu+\tau}{1+v+\tau}$ are plotted with pointwise credible intervals, bars are indicating the distribution of the observed covariate values, and again all other effects are kept constant. The nonlinear effects (centred around zero) on $\mu, v$ and $\sigma^{2}$ are depicted in Figure A19 together with pointwise credible intervals and bars again indicating the distribution of the observed covariate values. The raw centred posterior mean spatial effects on the four distribution parameters can be found in Figure A20, and estimates of linear effects on $\sigma^{2}$ and $\tau$ are given in Table $\mathrm{A} 2$

Findings on selected effects can be summarised as follows: Figure A17 indicates a gradient between the East of England and Wales or the West of England with higher production of cereal in the former one. This can be explained by the stony and 

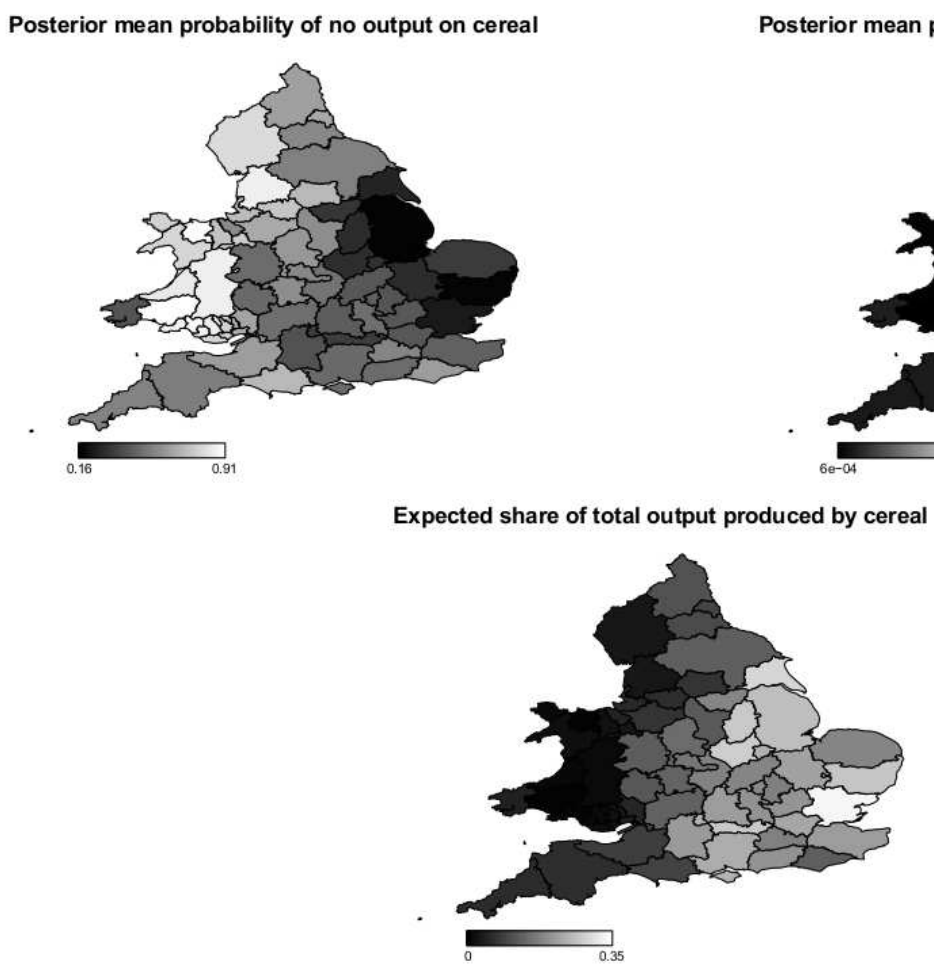

Figure A17: Cereal data. Posterior means for the probabilities $\frac{v}{1+v+\tau}$ (topleft), $\frac{\tau}{1+v+\tau}$ (topright) and posterior mean expected proportion $\frac{\mu+\tau}{1+v+\tau}$ of the total output on cereal (bottom) in the zero-one inflated beta model. The other effects are kept constant. Note that axes of subfigures have different ranges to enhance visibility of estimated effects. 

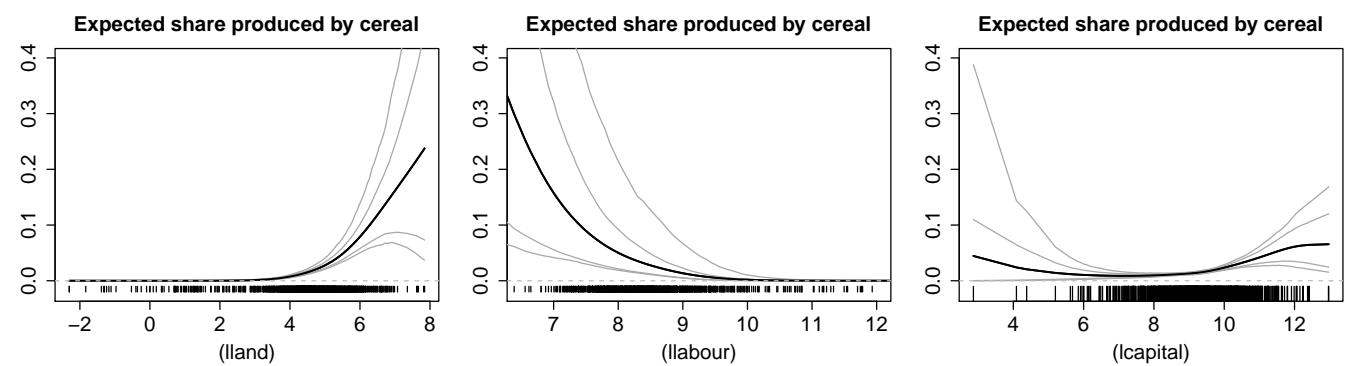

Figure A18: Cereal data. Posterior means for expected proportions $\frac{\mu+\tau}{1+v+\tau}$ for the production of cereal varying over lland (left), llabour (middle) and lcapital (right) together with $80 \%$ and $95 \%$ pointwise credible intervals in the zero-one inflated beta model. The other effects are kept constant.
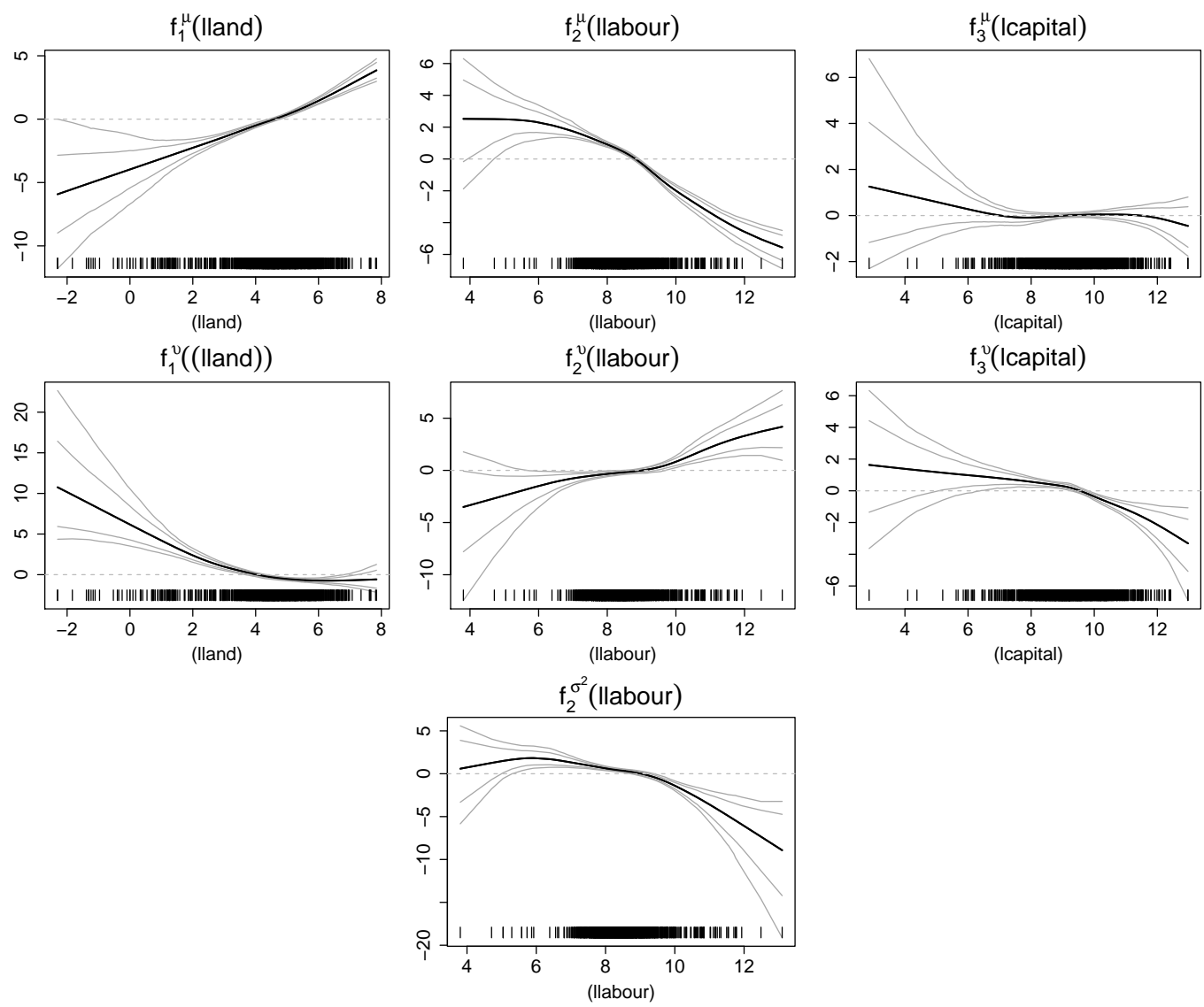

Figure A19: Cereal data. Posterior mean estimates of nonlinear effects on $\mu$ (first row), on $v$ (second row) and on $\sigma^{2}$ (third row) together with $80 \%$ and $95 \%$ pointwise credible intervals in the zero-one inflated beta model, centred around zero. Note that axes of subfigures have different ranges to enhance visibility of estimated effects.

hilly landscape of Wales and the coast in the East of England which is suited for the cultivation of cereal (compare also Figure A20). Figure A17 furthermore reveals that the probability of no cereal output is in general higher than the probability of obtaining a farm that produces only cereal. The total agricultural area of a farm is 

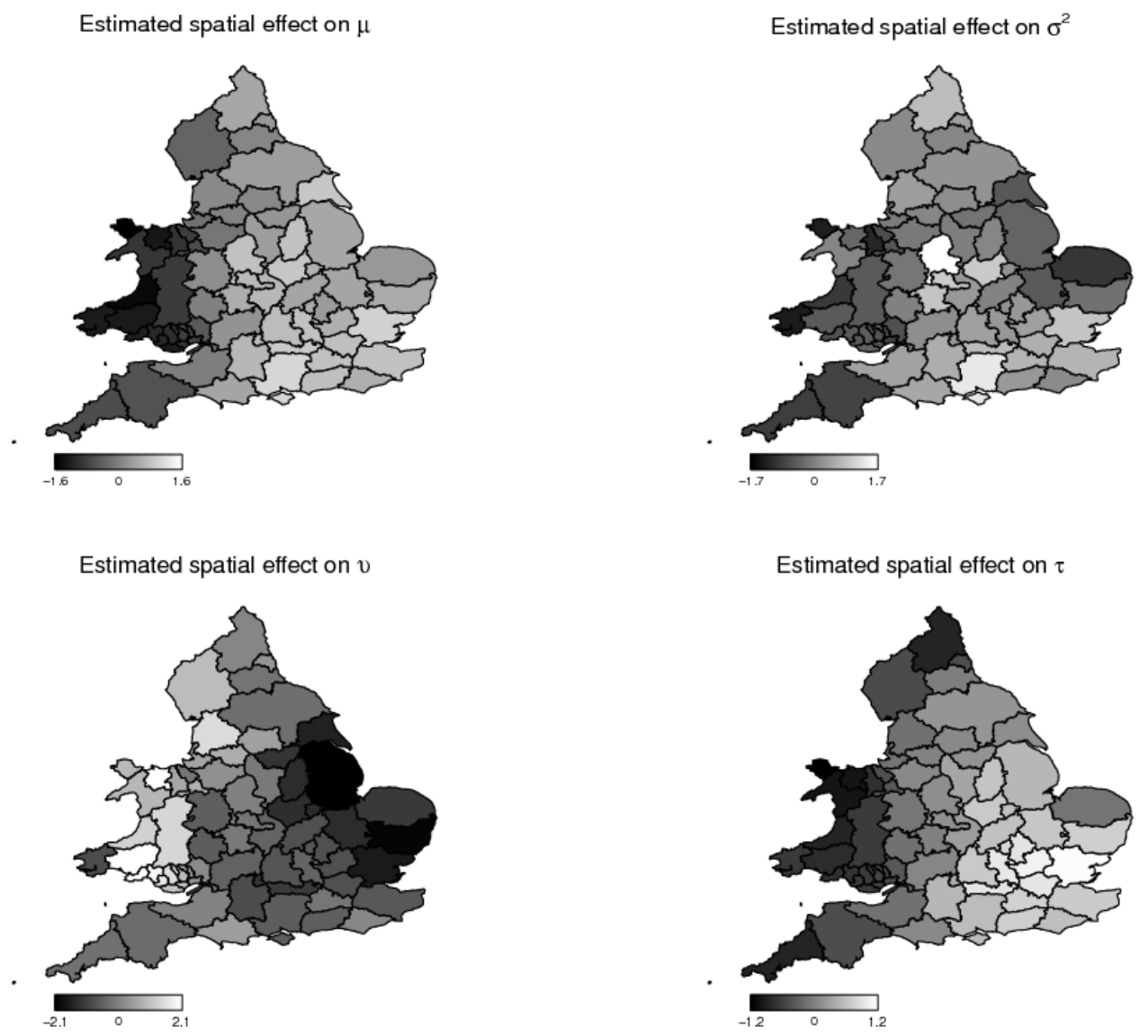

Figure A20: Cereal data. Posterior mean estimates of spatial effects in the zero-one inflated beta model, centred around zero. Note that axes of sub figures have different ranges to enhance visibility of estimated effects.

\begin{tabular}{l|cccc}
\hline \hline Parameter & mean & $2.5 \%$ quantile & median & $97.5 \%$ quantile \\
\hline$\beta_{0}^{\mu}$ (intercept) & -1.76 & -1.98 & -1.75 & -1.54 \\
$\beta_{0}^{\sigma^{2}}$ (intercept) & -5.52 & -7.36 & -5.3 & -3.61 \\
$\beta_{1}^{\sigma^{2}}$ (lland) & 0.41 & 0.14 & 0.41 & 0.68 \\
$\beta_{3}^{\sigma^{2}}$ (lcaptial) & 0.19 & -0.06 & 0.19 & 0.42 \\
$\beta_{0}^{v}$ (intercept) & 0.75 & 0.478 & 0.75 & 1.05 \\
$\beta_{0}^{\tau}$ (intercept) & 5.26 & 2.30 & 5.27 & 8.43 \\
$\beta_{1}^{\tau}$ (lland) & 1.02 & 0.58 & 1.02 & 1.45 \\
$\beta_{2}^{\tau}$ (llabour) & -1.53 & -2.01 & -1.53 & -1.08 \\
\hline \hline
\end{tabular}

Table A2: Cereal data. Summary of posterior distribution of linear effects in the zero-one inflated beta model.

estimated to be a crucial factor for or against a large production of cereals, compare Figure $\$ 19$ and $\beta_{1}^{\tau}$ in Table $\mathbf{A 2}$. For farms that do not have extreme capital compared 
to the remaining farms, the effect of capital is more or less insignificant. One possible explanation is the general problem of measuring capital in one quantity. Looking at the effects of labour it is important to be aware of the fact that e.g. a decreasing effect of $f_{2}^{\mu}$ in Figure A19 is not directly linked to the expected proportion of cereal products since human labour is also needed for the remaining output. However, the effect can be an indicator for the rising use of machineries and the aim to reduce costs in order to increase the efficiency of the farm. As in Figure A19, one percent increase of the nonlinear effects in Figure A18 has to be interpreted as one percent point increase of corresponding predictors respectively the expected proportion of cereal output.

\section{References}

Acemoglu, D. (2002). Technical change, inequality and the labor market, Journal of Economic Literature 40(1): 7-72.

Autor, D. H., Katz, L. F. and Kearney, M. S. (2008). Trends in U.S. wage inequality: Revising the revisionists, Review of Economics and Statistics 28: 300-323.

Bach, S., Corneo, G. and Steiner, V. (2009). From bottom to top: the entire income distribution in Germany, 1992-2003, Review of Income and Wealth 55: 303-330.

Ferrari, S. L. P. and Cribari-Neto, F. (2004). Beta regression for modelling rates and proportions, Journal of Applied Statistics 31: 799-815.

Statistisches Bundesamt (2012). Periodensterbetafeln für Deutschland: Allgemeine Sterbetafeln, abgekürzte Sterbetafeln und Sterbetafeln, Statistisches Bundesamt, Wiesbaden.

Wagner, G. G., Frick, J. R. and Schupp, J. (2007). The German socio-economic panel study (SOEP) - scope, evolution and enhancements, Schmollers Jahrbuch 127: 139-169.

Waldmann, E., Kneib, T., Yue, Y. R., Lang, S. and Flexeder, C. (2013). Bayesian semiparametric additive quantile regression, Statistical Modelling 13: 223-252. 